\title{
Efficacy and safety in a 4-year follow-up of the ELEVATE-TN study comparing acalabrutinib with or without obinutuzumab versus obinutuzumab plus chlorambucil in treatment-naïve chronic lymphocytic leukemia
}

\author{
Jeff P. Sharman $\mathbb{D}^{1 \times}$, Miklos Egyed ${ }^{2}$, Wojciech Jurczak (iD) ${ }^{3}$, Alan Skarbnik ${ }^{4}$, John M. Pagel (iD ${ }^{5}$, Ian W. Flinn (iD ${ }^{6}$, Manali Kamdar ${ }^{7}$, \\ Talha Munir ${ }^{8}$, Renata Walewska9 ${ }^{9}$, Gillian Corbett ${ }^{10}$, Laura Maria Fogliatto ${ }^{11}$, Yair Herishanu ${ }^{12}$, Versha Banerji ${ }^{13}$, Steven Coutre iD $^{14}$, \\ George Follows ${ }^{15}$, Patricia Walker ${ }^{16}$, Karin Karlsson ${ }^{17}$, Paolo Ghia (iD ${ }^{18}$, Ann Janssens ${ }^{19}$, Florence Cymbalista ${ }^{20}$, Jennifer A. Woyach (iD ${ }^{21}$, \\ Emmanuelle Ferrant ${ }^{22}$, William G. Wierda (iD ${ }^{23}$, Veerendra Munugalavadla ${ }^{24}$, Ting $\mathrm{Yu}^{24}$, Min Hui Wang ${ }^{24}$ and John C. Byrd ${ }^{21}$
}

(c) The Author(s) 2021

Leukemia (2022) 36:1171-1175; https://doi.org/10.1038/s41375-021-01485-x

\section{TO THE EDITOR}

Bruton tyrosine kinase (BTK) inhibitors have improved chronic lymphocytic leukemia (CLL) outcomes and offer a chemotherapyfree option [1]. The BTK inhibitor ibrutinib, alone or with a CD20 antibody, demonstrated better efficacy versus chemoimmunotherapy in treatment-naïve (TN) CLL [2-4]. However, cardiovascular toxicity is a concern with continuous ibrutinib use $[5,6]$.

Acalabrutinib is a next-generation, selective BTK inhibitor approved for CLL/small lymphocytic leukemia (SLL). Acalabrutinib, alone or with obinutuzumab, showed favorable efficacy in clinical trials [7, 8]. ELEVATE-TN demonstrated superior efficacy for acalabrutinib-obinutuzumab versus obinutuzumab-chlorambucil with acceptable tolerability in TN CLL [9]. We report 4-year followup results from ELEVATE-TN.

ELEVATE-TN is a phase 3 , randomized, multicenter, open-label study (NCT02475681) that enrolled patients aged $\geq 65$ years, or 18-65 years with comorbidities (Cumulative Illness Rating ScaleGeriatric score $>6$, creatinine clearance $30-69 \mathrm{~mL} / \mathrm{min}$ by Cockcroft-Gault), who had TN CLL or SLL requiring treatment, Eastern Cooperative Oncology Group performance status score of $\leq 2$, and adequate hematologic, hepatic, and renal function [9]. Patients were randomized (1:1:1) to acalabrutinib $100 \mathrm{mg}$ twice daily (until disease progression or unacceptable toxicity) with or without obinutuzumab (fixed-duration, up to 6 cycles) or obinutuzumab plus chlorambucil (up to 6 cycles). Crossover to acalabrutinib monotherapy was permitted in patients who progressed on obinutuzumab-chlorambucil. The primary study endpoint was independent review committee (IRC)-assessed progression-free survival (PFS). After primary analysis, PFS was investigatorassessed. Key secondary/exploratory endpoints were investigator-assessed PFS, investigator-assessed overall response rate (ORR), overall survival (OS), undetectable minimal residual disease (UMRD) rate, and safety. The study was not powered to compare acalabrutinib versus acalabrutinib-obinutuzumab. Informed consent was obtained from all patients before enrollment. Study details were previously published [9].

In total, 535 patients were randomized (acalabrutinib-obinutuzumab, $n=179$; acalabrutinib, $n=179$; obinutuzumab-chlorambucil, $n=177$ ). Median age was 70 years (range, $41.0-91.0$ ); $14 \%$ had del(17)(p13.1) and/or mutated TP53 and 63\% had unmutated immunoglobulin heavy chain variable (IGHV) gene (Supplementary Table 1).

At a median follow-up of 46.9 months (range, 0.0-59.4), treatment was ongoing in $74.9 \%(n=134)$ and $69.3 \%(n=124)$ of patients in the acalabrutinib-obinutuzumab and acalabrutinib monotherapy arms, respectively (Supplementary Table 2). Sixty-

\footnotetext{
${ }^{1}$ Willamette Valley Cancer Institute and Research Center, Eugene, OR, USA. ${ }^{2}$ Somogy County Mór Kaposi General Hospital, Kaposvár, Hungary. ${ }^{3}$ Maria Skłodowska-Curie National Research Institute of Oncology, Krakow, Poland. ${ }^{4}$ Novant Health Cancer Institute, Charlotte, NC, USA. ${ }^{5}$ Swedish Cancer Institute, Center for Blood Disorders and Stem Cell Transplantation, Seattle, WA, USA. ${ }^{6}$ Sarah Cannon Research Institute and Tennessee Oncology, Nashville, TN, USA. ${ }^{7}$ University of Colorado Cancer Center, Aurora, CO, USA. ${ }^{8}$ Haematology, Haematological Malignancy Diagnostic Service (HMDS), St. James's Institute of Oncology, Leeds, UK. ${ }^{9}$ Cancer Care, University Hospitals Dorset, Bournemouth, UK.

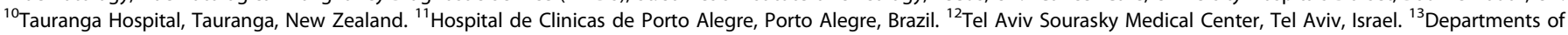
Internal Medicine, Biochemistry \& Medical Genetics, Max Rady College of Medicine, Rady Faculty of Health Sciences, University of Manitoba and CancerCare Manitoba Research Institute, Winnipeg, MB, Canada. ${ }^{14}$ Stanford University School of Medicine, Stanford, CA, USA. ${ }^{15}$ Department of Haematology, Addenbrooke's Hospital NHS Trust, Cambridge, UK. ${ }^{16}$ Peninsula Health and Peninsula Private Hospital, Frankston, Melbourne, VIC, Australia. ${ }^{17}$ Skåne University Hospital, Lund, Sweden. ${ }^{18}$ Università Vita-Salute San Raffaele and IRCCS Ospedale San Raffaele, Milano, Italy. ${ }^{19}$ University Hospitals Leuven, Leuven, Belgium. ${ }^{20}$ Bobigny: Hématologie, CHU Avicennes, Bobigny, France. ${ }^{21}$ The Ohio State University Comprehensive Cancer Center, Columbus, OH, USA. ${ }^{22}$ Hospices Civils de Lyon, Centre Hospitalier Lyon Sud, Service d'Hématologie Clinique, Pierre-Bénite, France. ${ }^{23}$ Department of Leukemia, Division of Cancer Medicine, MD Anderson Cancer Center, Houston, TX, USA. ${ }^{24}$ AstraZeneca, South San Francisco, CA, USA. ${ }^{\circledR}$ email: Jeff.Sharman@USONCOLOGY. COM
}

Received: 19 August 2021 Revised: 15 November 2021 Accepted: 17 November 2021

Published online: 1 January 2022 

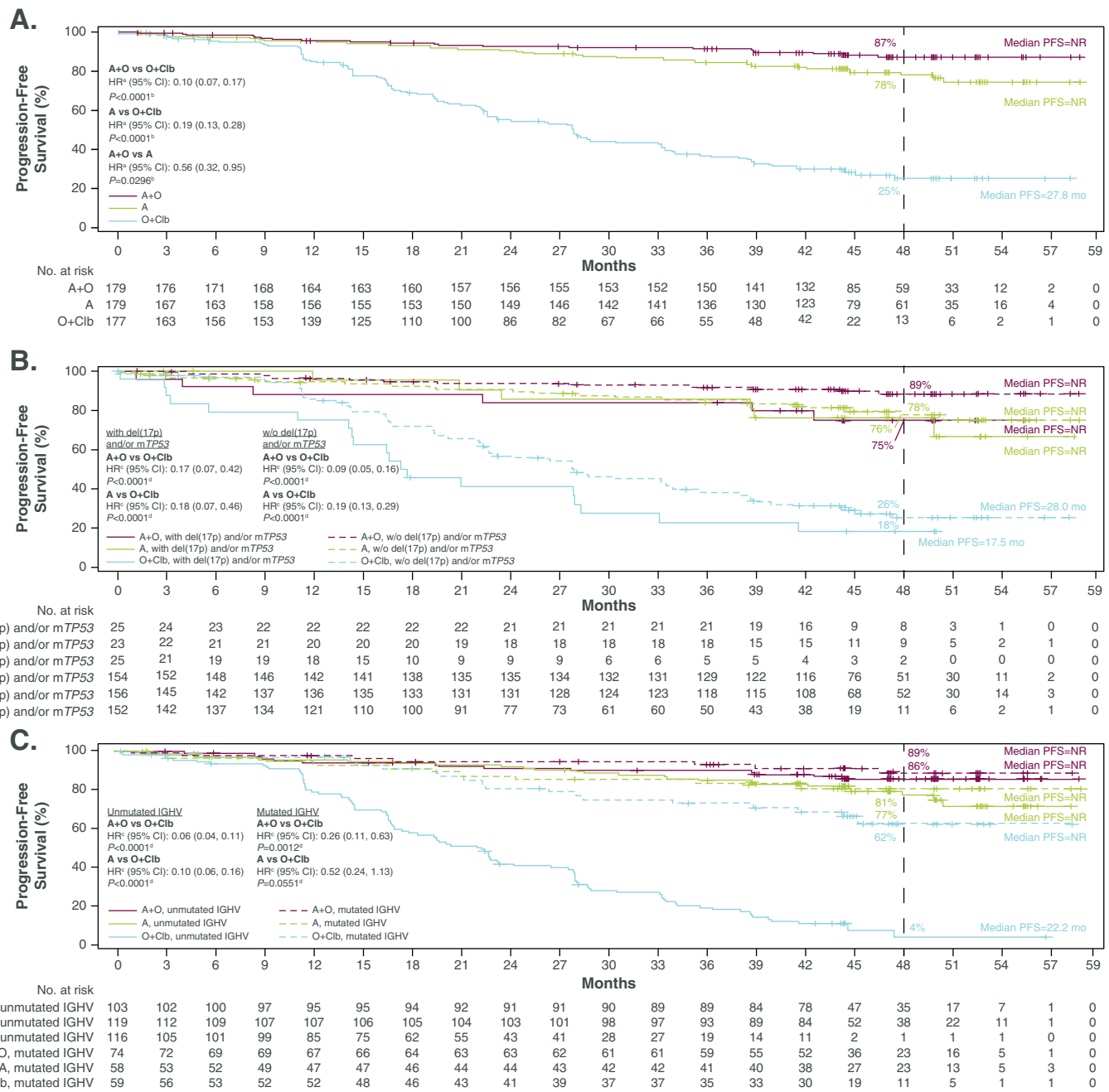

Fig. 1 Investigator-assessed progression-free survival (A) overall, (B) by del(17)(p13.1) and/or mutated TP53 status, and (C) by IGHV mutation status. ${ }^{a}$ Hazard ratio was based on stratified Cox-Proportional-Hazards model; ${ }^{b} P$ value was based on stratified log-rank test; ${ }^{C}$ Hazard ratio was based on unstratified Cox-Proportional-Hazards model. ${ }^{d} p$ value was based on unstratified log-rank test. A acalabrutinib, $\mathrm{Cl}$ confidence interval, Clb chlorambucil, HR hazard ratio, IGHV immunoglobulin heavy chain variable region, mTP53 mutated TP53, NR not reached, O obinutuzumab, PFS progression-free survival, w/o without.

nine patients (39.0\%) in the obinutuzumab-chlorambucil arm had crossed over to acalabrutinib. Overall, $25.1 \%$ of acalabrutinibobinutuzumab patients and $30.7 \%$ of acalabrutinib patients discontinued treatment; $22.6 \%$ of obinutuzumab-chlorambucil patients did not complete therapy. The most common reason for treatment discontinuation (acalabrutinib-obinutuzumab, acalabrutinib, and obinutuzumab-chlorambucil) was adverse events (AEs; $12.8 \%, 12.3 \%$, and $14.7 \%$, respectively).

Median investigator-assessed PFS was not reached (acalabrutinib-containing arms) versus 27.8 months for obinutuzumabchlorambucil (both $P<0.0001$; Fig. $1 \mathrm{~A}$ ). In a post hoc analysis, prolonged PFS also was observed with acalabrutinibobinutuzumab versus acalabrutinib ( $P=0.0296$; Fig. 1A); however, the study was not sufficiently powered for this comparison. The PFS benefit of acalabrutinib-containing regimens was consistent in high-risk genomic subgroups. In patients with del(17)(p13.1) and/ or mutated TP53, median PFS was not reached (acalabrutinibcontaining arms) versus 17.5 months for obinutuzumabchlorambucil (both $P<0.0001$; Fig. 1B); similar results were seen in patients with only del(17)(p13.1) (Supplementary Fig. 1). In patients with unmutated IGHV, median PFS was not reached (acalabrutinib-containing arms) versus 22.2 months for obinutuzumab-chlorambucil (both $P<0.0001$ ); median PFS was not reached in any treatment arm in patients with mutated IGHV (Fig. 1C). Estimated 48-month PFS rates overall were $87.0 \%$ for acalabrutinib-obinutuzumab, $77.9 \%$ for acalabrutinib, and $25.1 \%$ for obinutuzumab-chlorambucil. In the acalabrutinibobinutuzumab and acalabrutinib monotherapy arms, 48-month PFS rates were $74.8 \%$ and $76.2 \%$, respectively, for patients with del (17)(p13.1) and/or mutated TP53, and $85.7 \%$ and $77.1 \%$ for patients with unmutated IGHV.

Median OS was not reached in any treatment arm. Fewer deaths occurred in patients receiving acalabrutinib-obinutuzumab versus obinutuzumab-chlorambucil, but the difference was not statistically significant (HR: $0.50 ; 95 \% \quad C l, 0.25, \quad 1.02 ; P=0.0604$; Supplementary Fig. 2). While the OS HR for acalabrutinibobinutuzumab versus acalabrutinib in a post hoc analysis was noteworthy (HR: $0.53 ; 95 \% \mathrm{Cl}, 0.26,1.06$ ), the difference between the two acalabrutinib-containing arms was not statistically significant $(P=0.0836)$. Estimated 48-month OS rates were $92.9 \%$ for acalabrutinib-obinutuzumab, $87.6 \%$ for acalabrutinib, and $88.0 \%$ for obinutuzumab-chlorambucil.

The ORR was significantly higher with acalabrutinibobinutuzumab $(96.1 \%[n=172 / 179] ; 95 \% \mathrm{Cl}, 92.1,98.1)$ versus obinutuzumab-chlorambucil (82.5\% $[n=146 / 177]$; $95 \% \mathrm{Cl}, 76.2$, 87.4; $P<0.0001$; Supplementary Fig. 3A). The ORR with 
Table 1. Common adverse events (AEs) and selected AEs of interest.

\begin{tabular}{|c|c|c|c|c|c|c|}
\hline & \multicolumn{2}{|c|}{$A+O(n=178)$} & \multicolumn{2}{|l|}{$A(n=179)$} & \multicolumn{2}{|c|}{ O + Clb $(n=169)$} \\
\hline Treatment exposure, median (range), months & \multicolumn{2}{|c|}{$46.6(2.3-58.6)$} & \multicolumn{2}{|c|}{$45.7(0.3-59.3)$} & \multicolumn{2}{|l|}{$5.6(0.9-7.4)$} \\
\hline \multicolumn{7}{|c|}{ Common AEs (in $\geq 25 \%$ of patients [any grade] in any group), $n(\%)$} \\
\hline & Any grade & Grade $\geq 3$ & Any grade & Grade $\geq 3$ & Any grade & Grade $\geq 3$ \\
\hline Diarrhea & $73(41.0)$ & $9(5.1)$ & $72(40.2)$ & $1(0.6)$ & $36(21.3)$ & $3(1.8)$ \\
\hline Headache & $71(39.9)$ & $2(1.1)$ & $68(38.0)$ & $2(1.1)$ & $20(11.8)$ & 0 \\
\hline Neutropenia & $60(33.7)$ & $55(30.9)$ & $22(12.3)$ & $20(11.2)$ & $76(45.0)$ & $70(41.4)$ \\
\hline Arthralgia & $47(26.4)$ & $2(1.1)$ & 35 (19.6) & $2(1.1)$ & $8(4.7)$ & $2(1.2)$ \\
\hline Cough & $46(25.8)$ & $1(0.6)$ & $40(22.3)$ & $1(0.6)$ & $15(8.9)$ & 0 \\
\hline URTI & $44(24.7)$ & $4(2.2)$ & $46(25.7)$ & 0 & $16(9.5)$ & $1(0.6)$ \\
\hline Nausea & $41(23.0)$ & 0 & 41 (22.9) & 0 & $53(31.4)$ & 0 \\
\hline IRR & $25(14.0)$ & $5(2.8)$ & 0 & 0 & $68(40.2)$ & $10(5.9)$ \\
\hline Major bleeding ${ }^{d}$ & 7 (3.9) & $5(2.8)$ & 7 (3.9) & $5(2.8)$ & $2(1.2)$ & 0 \\
\hline Hypertension & $14(7.9)$ & $6(3.4)$ & $13(7.3)$ & $5(2.8)$ & $7(4.1)$ & $6(3.6)$ \\
\hline Infections & $134(75.3)$ & $42(23.6)$ & $132(73.7)$ & $29(16.2)$ & $75(44.4)$ & $14(8.3)$ \\
\hline SPMs & $28(15.7)$ & $13(7.3)$ & $24(13.4)$ & $5(2.8)$ & $7(4.1)$ & $3(1.8)$ \\
\hline Excluding NMS & $15(8.4)$ & $10(5.6)$ & $11(6.1)$ & $4(2.2)$ & $3(1.8)$ & $2(1.2)$ \\
\hline
\end{tabular}

$A$ acalabrutinib, $A E$ adverse event, Clb chlorambucil, IRR infusion-related reaction, NMS non-melanoma skin, $O$ obinutuzumab, SPMs secondary primary malignancies, URTI upper respiratory tract infection.

${ }^{a}$ Cardiac events that occurred in $>1$ patient (any grade; other than atrial fibrillation) in any group include angina pectoris, palpitations, atrioventricular block complete, myocardial ischemia, tachycardia, bradycardia, cardiac failure, left ventricular failure, myocardial infarction, pericardial effusion, acute myocardial infarction, and supraventricular tachycardia.

${ }^{\mathrm{b}}$ Cardiac events (grade $\geq 3$ ) that occurred in $>1$ patient (other than atrial fibrillation) include atrioventricular block complete $(n=3)$, angina pectoris $(n=2)$, myocardial ischemia $(n=2)$, and myocardial infarction $(n=2)$.

${ }^{c}$ Cardiac events (grade $\geq 3$ ) that occurred in $>1$ patient (other than atrial fibrillation) include acute myocardial infarction ( $n=3$ ), cardiac failure ( $\left.n=2\right)$, and myocardial infarction $(n=2)$.

${ }^{d}$ Defined as any serious or grade $\geq 3$ hemorrhagic event, or any-grade hemorrhagic event in the central nervous system.

acalabrutinib (89.9\% $[n=161 / 179] ; 95 \% \mathrm{Cl}, 84.7,93.5)$ also was significantly higher versus obinutuzumab-chlorambucil $(P=$ 0.035). The complete response (CR) rate, including $C R$ with incomplete hematologic recovery (CRi), was higher with acalabrutinib-obinutuzumab $\quad(30.7 \% \quad[n=55 / 179]) \quad$ versus obinutuzumab-chlorambucil $(13.0 \%[n=23 / 177])$ and versus acalabrutinib (post hoc; $11.2 \% \quad[n=20 / 179]$ ). Comparing the acalabrutinib-obinutuzumab and acalabrutinib monotherapy arms, CR + CRi rates were $32.0 \%$ and $13.0 \%$, respectively, for patients with del(17)(p13.1) and/or mutated TP53, and 28.2\% and $12.6 \%$ for patients with unmutated IGHV. Sustained UMRD rates based on the last two MRD assessments are shown in Supplementary Fig. 3B.

Median treatment exposure was $\mathbf{4 6 . 6}$ months for acalabrutinibobinutuzumab and 45.7 months for acalabrutinib monotherapy (Table 1); no new safety signals were observed. The most common any-grade AEs ( $\geq 30 \%$ ) were diarrhea, headache, and neutropenia for acalabrutinib-obinutuzumab; diarrhea and headache for acalabrutinib monotherapy; and neutropenia, infusion-related reaction, and nausea for obinutuzumab-chlorambucil (Table 1). AEs occurring more frequently in the acalabrutinib-containing arms included headache, diarrhea, fatigue, arthralgia, cough, and upper respiratory tract infection. Headaches, while common, were typically low grade; none led to treatment discontinuation. Among patients receiving acalabrutinib-obinutuzumab, neutropenia, fatigue, and arthralgia were more frequent relative to acalabrutinib alone. The obinutuzumab-chlorambucil arm had more frequent neutropenia, nausea, and infusion-related reactions relative to both acalabrutinib-containing arms, though differences in $\mathrm{AE}$ reporting could be due to the longer treatment exposure in the acalabrutinib-containing arms versus the comparator arm. In the acalabrutinib-containing arms, most of the common AEs decreased in incidence over time, and most events occurred more predominantly during the first year of treatment (Supplementary Table 3). Incidence and time to onset of AEs leading to discontinuation of acalabrutinib-containing treatment are described in Supplementary Table 4.

Events of clinical interest (ECls), including cardiovascular events, were similar in both acalabrutinib arms (Table 1). In addition, the cumulative incidences of atrial fibrillation and hypertension over time were low and similar across treatment groups (Supplementary Fig. 4).

With a median follow-up of 46.9 months, the efficacy and safety of acalabrutinib plus obinutuzumab and acalabrutinib monotherapy were maintained with low rates of treatment discontinuation. Median PFS was not reached for either acalabrutinib-containing arm, and PFS continued to be significantly longer for both acalabrutinib-containing arms versus obinutuzumab-chlorambucil. Consistent with the primary report [9], the acalabrutinibcontaining arms continued to demonstrate significantly greater PFS benefits versus obinutuzumab-chlorambucil in high-risk genomic subgroups, including $\operatorname{del}(17)(\mathrm{p} 13.1)$ and/or mutated 
TP53 and unmutated IGHV, with longer-term treatment. Of note, the estimated PFS rate at 48 months trended in favor of the acalabrutinib combination versus acalabrutinib monotherapy, consistent with findings from preclinical studies demonstrating that, in contrast to ibrutinib, acalabrutinib does not interfere with the anti-tumor immune-mediated mechanisms of anti-CD20 monoclonal antibodies [10, 11]. In the acalabrutinib-containing arms, the CR/CRi rate increased from the primary analysis at 28.3 months (acalabrutinib-obinutuzumab: 24.0\%; acalabrutinib: $7.8 \%$ [9]) to the current report at a follow-up of 4 years $(30.7 \%$ and $11.2 \%$, respectively). In high-risk subgroups, CR/CRi rates were numerically higher with the acalabrutinib combination versus monotherapy; however, the study was not powered for this comparison. Further research is needed to assess the efficacy benefits of acalabrutinib-obinutuzumab combination therapy. With longer-term follow-up, the tolerability profile of the acalabrutinib-containing arms was consistent with that of the primary analysis. Incidences of the most common AEs, such as headache, diarrhea, neutropenia, and fatigue, were generally unchanged or saw a slight increase from the primary analysis [9].

Though cross-trial comparisons are limited, the efficacy results from this study are aligned with those from the iLLUMINATE study of ibrutinib-obinutuzumab in a similar patient population at a median follow-up of 31.3 months [4]. In that study, median PFS (assessed by IRC) was not reached; the estimated 30-month PFS rate was $79 \%$ with ibrutinib-obinutuzumab. Atrial fibrillation and hypertension rates with ibrutinib-obinutuzumab (12 and 17\%, respectively) in iLLUMINATE [4] were higher than the atrial fibrillation/flutter and hypertension rates reported with acalabrutinib-obinutuzumab in the present study (4 and $8 \%$ ). Discontinuation due to AEs was similar with ibrutinibobinutuzumab (16\%) in the iLLUMINATE study and with acalabrutinib-obinutuzumab in the present study (13\%). By comparison, a head-to-head study of acalabrutinib versus ibrutinib (ELEVATE-RR; NCT02477696) at a median follow-up of 40.9 months demonstrated non-inferiority for PFS (primary endpoint) and a statistically significantly lower incidence of atrial fibrillation/flutter with acalabrutinib versus ibrutinib ( $9 \%$ vs $16 \%$, respectively) in patients with previously treated CLL [12]. In ELEVATE-RR, hypertension incidence was also statistically higher with ibrutinib versus acalabrutinib ( $23 \%$ vs $9 \%$ ).

Based on these updated results, ELEVATE-TN shows continued efficacy at 4 years and a significant PFS benefit in the acalabrutinib-containing arms regardless of high-risk status. PFS benefit is seen particularly with acalabrutinib-obinutuzumab, although this combination resulted in a higher incidence of AEs compared with acalabrutinib monotherapy. No new safety signals were observed with acalabrutinib-containing treatment with longer-term follow-up. The safety of acalabrutinib-containing treatment was consistent with the primary analysis [9], with a low incidence of ECls, particularly cardiovascular AEs (atrial fibrillation/flutter and hypertension) and low rates of treatment discontinuation despite longer treatment exposure. Findings illustrate the flexibility to tailor acalabrutinib treatment as monotherapy or combination treatment and support acalabrutinib as a combination partner with obinutuzumab in the first-line CLL setting.

\section{REFERENCES}

1. Wen T, Wang J, Shi Y, Qian H, Liu P. Inhibitors targeting Bruton's tyrosine kinase in cancers: drug development advances. Leukemia. 2021;35:312-32.

2. Woyach JA, Ruppert AS, Heerema NA, Zhao W, Booth AM, Ding W, et al. Ibrutinib regimens versus chemoimmunotherapy in older patients with untreated CLL. N Engl J Med. 2018;379:2517-28.

3. Shanafelt TD, Wang XV, Kay NE, Hanson CA, O'Brien S, Barrientos J, et al. Ibrutinibrituximab or chemoimmunotherapy for chronic lymphocytic leukemia. N Engl J Med. 2019;381:432-43.
4. Moreno C, Greil R, Demirkan F, Tedeschi A, Anz B, Larratt L, et al. Ibrutinib plus obinutuzumab versus chlorambucil plus obinutuzumab in first-line treatment of chronic lymphocytic leukaemia (iLLUMINATE): a multicentre, randomised, openlabel, phase 3 trial. Lancet Oncol. 2019;20:43-56.

5. Pellegrini L, Novak U, Andres M, Suter T, Nagler M. Risk of bleeding complications and atrial fibrillation associated with ibrutinib treatment: a systematic review and meta-analysis. Crit Rev Oncol Hematol. 2021;159:103238.

6. Archibald WJ, Rabe KG, Kabat BF, Herrmann J, Ding W, Kay NE, et al. Atrial fibrillation in patients with chronic lymphocytic leukemia (CLL) treated with ibrutinib: risk prediction, management, and clinical outcomes. Ann Hematol. 2021;100:143-55.

7. Byrd JC, Woyach JA, Furman RR, Martin P, O'Brien S, Brown JR, et al. Acalabrutinib in treatment-naïve chronic lymphocytic leukemia. Blood. 2021;137:3327-38.

8. Woyach JA, Blachly JS, Rogers KA, Bhat SA, Jianfar M, Lozanski G, et al. Acalabrutinib plus obinutuzumab in treatment-naive and relapsed/refractory chronic lymphocytic leukemia. Cancer Disco. 2020;10:394-405.

9. Sharman JP, Egyed M, Jurczak W, Skarbnik A, Pagel JM, Kamdar M, et al. Acalabrutinib with or without obinutuzumab versus chlorambucil and obinutuzmab for treatment-naive chronic lymphocytic leukaemia (ELEVATE TN): a randomised, controlled, phase 3 trial. Lancet. 2020;395:1278-91.

10. Golay J, Ubiali G, Introna M. The specific Bruton tyrosine kinase inhibitor acalabrutinib (ACP-196) shows favorable in vitro activity against chronic lymphocytic leukemia B cells with CD20 antibodies. Haematologica. 2017;102:e400-e403.

11. Chu CC, Pinney JJ, Blick-Nitko SK, Baran AM, Peterson DR, Whitehead HE, et al. Ibrutinib off-target inhibition inhibits antibody-dependent cellular phagocytosis but not efferocytosis of CLL cells [abstract]. Blood. 2020;136 Suppl 1:45.

12. Byrd JC, Hillmen P, Ghia P, Kater AP, Chanan-Khan A, Furman RR, et al. Acalabrutinib versus ibrutinib in previously treated chronic lymphocytic leukemia: results of the first randomized phase III trial. J Clin Oncol. 2021;39:3441-52.

\section{ACKNOWLEDGEMENTS}

The authors are grateful for the contributions of our co-author, colleague, and friend Dr. Steven Coutre, who sadly passed away just before publication of this report; he will be dearly missed. The study was funded by Acerta Pharma, South San Francisco, CA, a member of the AstraZeneca Group. Medical writing assistance, funded by AstraZeneca, was provided by Tracy Diaz, PhD, and Cindy Gobbel, PhD, of Peloton Advantage, LLC, an OPEN Health company, Parsippany, NJ.

\section{AUTHOR CONTRIBUTIONS}

All authors contributed to data interpretation. Statistical analyses were performed by MHW. All authors reviewed and provided important intellectual contributions to the manuscript; all authors approved the final version for publication.

\section{COMPETING INTERESTS}

JS: Research funding from Seattle Genetics; research funding and consulting from AbbVie, AstraZeneca, BeiGene, BMS, Genentech, Gilead, Lilly, Pharmacyclics, TG Therapeutics; scientific board for Centessa; employment from US Oncology. ME: Nothing to disclose. WJ: Research funding from AstraZeneca, Janssen, and Loxo. AS: Consulting fees from AbbVie, AstraZeneca, Celgene, Genentech, GenMab, Kite, MorphoSys, Novartis, Pharmacyclics, Janssen, Alexion, Seattle Genetics, and TG Therapeutics; honoraria from AbbVie, AstraZeneca, BeiGene, Celgene, Genentech, Jazz Pharmaceuticals, Kite, Seattle Genetics, Pharmacyclics, Janssen, and TG Therapeutics; data safety monitoring board/advisory board participation for Alexion. JP: Consulting and speaker bureau for AstraZeneca. IF: Consulting fees from AbbVie, AstraZeneca, BeiGene, Genentech, Gilead, Great Point Partners, Iksuda, Janssen, Juno, Kite, MorphoSys, Nurix, Pharmacyclics, Roche, Seattle Genetics, Takeda, TG Therapeutics, Unum, Verastem, and Yingli; grants from AbbVie, Acerta, Agios, ArQule, AstraZeneca, BeiGene, Calithera, Celgene, Constellation, Curis, Forma, Forty Seven, Genentech, Gilead, IGM Biosciences, Incyte, Infinity, Janssen, Juno, Karyopharm, Kite, Loxo, Merck, MorphoSys, Novartis, Pfizer, Pharmacyclics, Portola, Rhizen, Roche, Seattle Genetics, Takeda, Teva, TG Therapeutics, Trilium, Triphase, Unum, and Verastem. MK: Research support/funding from TG Therapeutics and Genentech; consulting for AbbVie, Karyopharm, Kite, AstraZeneca, Celgene/Bristol Myers Squibb, Adaptive, and ADC Therapeutics; speaker's bureau for Seagen; data monitoring committee member for Celgene and Genentech. TM: Consulting/advisory role for Morphosys, Sunesis, AstraZeneca, Janssen; honoraria from Janssen, AstraZeneca, Gilead, and Novartis. RW: Nothing to disclose. GC: Nothing to disclose. LMF: Nothing to disclose. YH: Honoraria from AbbVie, Janssen, AstraZeneca, and Roche. VB: Honoraria from AbbVie, Janssen, and AstraZeneca; grant funding from the Canadian Institutes of Health Research, Leukemia Lymphoma Society of Canada, CancerCare Manitoba Foundation, and the 
Hairy Cell Leukemia Foundation. SC: Honoraria from Janssen Oncology and Pharmacyclics; consulting for AbbVie, Adaptive Biotechnologies, AstraZeneca, BeiGene, Genentech/Roche, Janssen Oncology, and Pharmacyclics; clinical research support from AbbVie, Acerta Pharma/AstraZeneca, Janssen Oncology, Pharmacyclics; expert testimony for Genentech. GF: Consulting fees from AstraZeneca, Roche, AbbVie, Janssen, Karyopharm, Bristol Myers Squibb, and Centessa; honoraria from AstraZeneca, Roche, AbbVie, and Janssen; travel support from Roche, Janssen, and Bristol Myers Squibb. PW: Travel support from Roche; honoraria from AstraZeneca. KK: Nothing to disclose. PG: Honoraria from AbbVie, AstraZeneca, ArQule/MSD, BeiGene, Janssen, Loxo/Lilly, and Roche; research support from AbbVie, AstraZeneca, Janssen, and Sunesis. AJ: Consulting fees from Janssen, Roche, Gilead, AbbVie, Novartis, Amgen, Sanofi-Genzyme, and AstraZeneca; honoraria from Janssen, Roche, AbbVie, Novartis, Amgen, AstraZeneca, and BeiGene; travel support from AbbVie, Roche, Amgen, and Bristol Myers Squibb/Celgene. FC: Consulting fees from AbbVie; honoraria from Roche, AbbVie, AstraZeneca, and Janssen; travel support from AstraZeneca, Roche, and AbbVie. JW: Consulting for AbbVie, Janssen, Pharmacyclics, BeiGene, AstraZeneca, ArQule, and Loxo; research support from Loxo and AbbVie; clinical trial support from Karyopharm, Morphosys, Pharmacyclics, and Janssen. EF: Honoraria from Janssen-Cilag; payment for expert testimony from Gilead, AbbVie, and Janssen-Cilag. WW: Grants/ contracts from GSK/Novartis, AbbVie, Genentech, Pharmacyclics, AstraZeneca/Acerta, Gilead, Juno, Kite, Sunesis, Miragen, Oncternal, Cyclacel, Loxo, Janssen, and Xencor. VM: Stock from and employment by AstraZeneca; family member association with Gilead. TY: Employment by AstraZeneca; stock from AstraZeneca, Johnson \& Johnson, and AbbVie. MHW: Stock from and employment by AstraZeneca. JB: Stock and other ownership interests from Vincerx; honoraria from Pharmacyclics, AstraZeneca, Novartis, Syndax, and Trillium; consulting or advisory role for Acerta, Janssen, Kura, Novartis, Syndax, and AstraZeneca; research funding from Acerta, Pharmacyclics, and Zencor; patents, royalties, and other intellectual property from OSU Patents; travel support from Gilead, Janssen, Novartis, Pharmacyclics, and TG Therapeutics.
ADDITIONAL INFORMATION

Supplementary information The online version contains supplementary material available at https://doi.org/10.1038/s41375-021-01485-x.

Correspondence and requests for materials should be addressed to Jeff P. Sharman.

Reprints and permission information is available at http://www.nature.com/ reprints

Publisher's note Springer Nature remains neutral with regard to jurisdictional claims in published maps and institutional affiliations.

\begin{abstract}
(c) (i)
Open Access This article is licensed under a Creative Commons Attribution 4.0 International License, which permits use, sharing, adaptation, distribution and reproduction in any medium or format, as long as you give appropriate credit to the original author(s) and the source, provide a link to the Creative Commons license, and indicate if changes were made. The images or other third party material in this article are included in the article's Creative Commons license, unless indicated otherwise in a credit line to the material. If material is not included in the article's Creative Commons license and your intended use is not permitted by statutory regulation or exceeds the permitted use, you will need to obtain permission directly from the copyright holder. To view a copy of this license, visit http://creativecommons. org/licenses/by/4.0/.
\end{abstract}

(c) The Author(s) 2021 